PROCEEDINGS OF THE AMERICAN MATHEMATICAL SOCIETY

Volume 124, Number 11, November 1996

\title{
ON THE RANGE OF THE SUM OF MONOTONE OPERATORS IN GENERAL BANACH SPACES
}

\author{
HASSAN RIAHI
}

(Communicated by Palle E. T. Jorgensen)

\begin{abstract}
The purpose of this paper is to generalize the Brézis-Haraux theorem on the range of the sum of monotone operators from a Hilbert space to general Banach spaces. The result obtained provides that the range $\mathcal{R}\left(\overline{A+B^{\tau}}\right)$ is topologically almost equal to the $\operatorname{sum} \mathcal{R}(A)+\mathcal{R}(B)$ where $\tau$ is a compatible topology in $X^{* *} \times X^{*}$ as proposed by Gossez. To illustrate the main result we consider some basic properties of densely maximal monotone operators.
\end{abstract}

\section{INTRODUCTION AND BACKGROUND}

The Brézis-Haraux theorem [3] on the range of the sum of monotone operators on a Hilbert space is an efficient tool in nonlinear analysis. Nevertheless, the exploration for this direction has continued its development also in a Hilbert space to nonmonotone linear operators that have been obtained by Brézis and Nirenberg [4]. In restrictive Banach spaces ( $L^{p}$ with $1<p<+\infty$ and reflexive) for accretive and $\phi$-monotone operators, some of these results have been obtained by Browder [5], Gupta and Hess [11], Calvert and Gupta [7], Reich [15],... In a recent paper, Morales [13] extended these results to some regular Banach spaces (uniformly convex) for $m$-accretive operators. Most applications that prove the existence of solutions for nonlinear partial differential equations, differential inclusions, optimization, calculus of variations,... have been proposed in the references quoted above (see, for instance, [17]).

The developed theory of monotone operators from a Banach space to its dual space can be considered most naturally as an extension of the fundamental theory of monotone operators on Hilbert spaces as was studied by Minty (1962) and Browder (1963). Another treatment is to consider accretive operators from a Banach space to itself. Naturally, in a Hilbert space one has the fundamental equivalence between maximal monotonicity and $m$-accretivity.

The purpose of this paper is to treat the range of the sum of monotone operators. The result obtained generalizes the Brézis-Haraux theorem from a Hilbert space to general Banach spaces by dealing with monotone operators. We derive in a more general Banach space $X$ the relations $\overline{\mathcal{R}(A+B)}=\overline{\mathcal{R}(A)+\mathcal{R}(B)}$ and $\operatorname{int}(\mathcal{R}(A+B)) \subset \operatorname{int}(\mathcal{R}(A)+\mathcal{R}(B)) \subset \operatorname{int}\left(\mathcal{R}\left(\overline{A+B^{\tau}}\right)\right)$ where $\tau$ is a compatible

Received by the editors April 18, 1994 and, in revised form, January 31, 1995.

1991 Mathematics Subject Classification. Primary 47H05; Secondary 46B10, 35J60.

Key words and phrases. Banach space, densely maximal monotone operator, $3^{*}$-monotone operator, range, subdifferential. 
topology in $X^{* *} \times X^{*}$ as proposed by Gossez. To illustrate the main result we consider some basic properties of densely maximal monotone operators (see [9], [14]). It is important to mention that these results can be applied to various problems in nonlinear analysis; especially for studying nonlinear differential inclusions and the Hammerstein integral equation.

Let $X$ be a real Banach space, let $X^{*}$ be its topological dual space and let $X^{* *}$ be its bidual space. The generalized duality pairing is denoted by $\langle.,$.$\left.\rangle (resp. \langle., .\rangle_{*}\right)$ between $X$ and $X^{*}$ (resp. $X^{*}$ and $X^{* *}$ ). We use $\operatorname{cl}_{\nu}(C)$ and $\operatorname{int}_{\nu}(C)$ to denote, respectively, the closure and the interior of a subset $C$ of $X$ relative to the topology $\nu$. When $\nu$ is the strong (norm) topology, we write $\operatorname{cl}_{\nu} C=\bar{C}$ and $\operatorname{int}_{\nu}(C)=\operatorname{int}(C)$. We also use $a+\tau B$ to denote the open ball centered at $a \in X$ with radius $r>0$.

By identifying the space $X$ with a subspace of $X^{* *}$, we see that $\operatorname{cl}_{\tau_{1}} X=X^{* *}$, where $\tau_{1}$ is the least fine topology for which the following mappings are continuous:

$$
\begin{aligned}
& X^{* *} \rightarrow \mathbb{R}: x^{* *} \rightarrow\left\langle x^{* *}, x^{*}\right\rangle_{*}, \quad \forall x^{*} \in X^{*}, \\
& X^{* *} \rightarrow \mathbb{R}: x^{* *} \rightarrow\left\|x^{* *}\right\| .
\end{aligned}
$$

Let $\tau=\tau_{1} \otimes \tau_{s}$ where $\tau_{s}$ is the strong topology on $X^{*}$.

An operator $A: X \rightarrow 2^{X^{*}}$ is a multivalued mapping from $X$ to $2^{X^{*}}$. Its domain and range are denoted by $\mathcal{D}(A)=\{x \in X: A x \neq \emptyset\}$ and $\mathcal{R}(A)=\left\{x^{*} \in X^{*}: x^{*} \in\right.$ $A x, x \in \mathcal{D}(A)\}$. Throughout this paper we identify an operator $A$ with its graph, i.e.

$$
x^{*} \in A x \Leftrightarrow\left(x, x^{*}\right) \in A \Leftrightarrow x \in A^{-1} x^{*} .
$$

We say that an operator $A: X \rightarrow 2^{X^{*}}$ is monotone if

$$
\forall\left(x_{1}, y_{1}\right) \in A, \forall\left(x_{2}, y_{2}\right) \in A \quad\left\langle y_{2}-y_{1}, x_{2}-x_{1}\right\rangle \geq 0 .
$$

It is said to be maximal monotone if its graph is maximal in the family of monotone operators in $X \times X^{*}$, ordered by inclusion.

We say that an operator $A: X \rightarrow 2^{X^{*}}$ is densely maximal if there exists a maximal monotone operator $B: X^{* *} \rightarrow 2^{X^{*}}$ such that $B \subset \bar{A}^{\tau}$ where

$$
\bar{A}^{\tau}=\operatorname{cl}_{\tau} A:=\left\{\left(x^{* *}, x^{*}\right) \in X^{* *} \times X^{*} ; \exists\left(x_{i}, x_{i}^{*}\right) \in A \text { with }\left(x_{i}, x_{i}^{*}\right) \stackrel{\tau}{\rightarrow}\left(x^{* *}, x^{*}\right)\right\} .
$$

The operator $\bar{A}^{\tau}$ need not be monotone in $X^{* *} \times X^{*}$ (see [10]). If $A$ is monotone, the dense maximality can be expressed by $\bar{A}^{\tau}$ is maximal monotone in $X^{* *} \times X^{*}$.

We now define the normalized duality mapping $J: X \rightarrow 2^{X^{*}}$ by

$$
J(x)=\left\{x^{*} \in X^{*} ;\left\langle x^{*}, x\right\rangle=\|x\|^{2},\left\|x^{*}\right\|=\|x\|\right\} .
$$

The $\varepsilon$-normalized duality mapping $J_{\varepsilon}: X \rightarrow 2^{X^{*}}$ is a multivalued operator defined by

$$
J_{\varepsilon}(x)=\left\{x^{*} \in X^{*} ; \frac{1}{2}\left(\|x\|^{2}+\left\|x^{*}\right\|^{2}\right) \leq\left\langle x^{*}, x\right\rangle+\varepsilon\right\} .
$$

Given $f: X \rightarrow \mathbb{R} \cup\{+\infty\}$ convex, lower semicontinuous and proper, its effective domain is given by $\operatorname{dom}(f)=\{x \in X / f(x)<+\infty\}$. The subdifferential (resp. $\varepsilon$-subdifferential) operator $\partial f$ (resp. $\left.\partial_{\varepsilon} f\right): X \rightarrow 2^{X^{*}}$ is defined by

$$
y \in \partial f(x) \quad \text { iff } \quad f^{*}(y)+f(x)=\langle y, x\rangle
$$

(resp.

$$
\left.y \in \partial_{\varepsilon} f(x) \quad \text { iff } \quad f(v) \geq f(x)+\langle y, v-x\rangle-\varepsilon \text { for all } v \text { in } X\right),
$$

where $f^{*}(y)=\sup _{u \in X}(\langle y, u\rangle-f(u))$ is the Legendre-Fenchel conjugate of $f$. 
The operator $\partial f \subset X \times X^{*}$ is maximal monotone (see [16]). It is also densely maximal (see [8, Théorème 3.1]). It follows from these definitions that

$$
J(x)=\partial\left(\frac{1}{2}\|\cdot\|^{2}\right)(x) \quad \text { and } \quad J_{\varepsilon}(x)=\partial_{\varepsilon}\left(\frac{1}{2}\|\cdot\|^{2}\right)(x) .
$$

The inf-convolution (also called epigraphic sum) of $f$ and $g$ is given by

$$
f \stackrel{e}{+} g(x)=\inf _{u \in X}(f(u)+g(x-u))
$$

\section{The MAIN RESUlts}

Definition (Aubin and Ekeland [2, p. 393], Zeidler [17, p. 901]). Let $A$ be a monotone operator from a Banach space $X$ to its dual $X^{*}$. Then $A$ is said to be $3^{*}$-monotone if for all $x^{*} \in \mathcal{R}(A)$ and $x \in \mathcal{D}(A)$ there is a real number $\alpha$ such that

$$
\inf _{(u, v) \in A}\left\langle x^{*}-v, x-u\right\rangle \geq \alpha .
$$

Lemma 1. (a) The operator $J_{\varepsilon}: X \rightarrow 2^{X^{*}}$ is bounded and coercive, i.e.

$$
\inf _{x^{*} \in J_{\varepsilon}(x)} \frac{\left\langle x^{*}, x\right\rangle}{\|x\|}=+\infty .
$$

(b) If $x^{*} \in J_{\varepsilon}(x)$, then $\left|\|x\|-\left\|x^{*}\right\|\right| \leq \sqrt{2 \varepsilon}$ and $\|x\| \cdot\left\|x^{*}\right\|-\varepsilon \leq\left\langle x^{*}, x\right\rangle$.

Proof. For (a) see [8, Lemme 1.3]. To show (b) it suffices to use the fact $J_{\varepsilon}(x)=$ $\partial_{\varepsilon}\left(\frac{1}{2}\|\cdot\|^{2}\right)(x)$.

Lemma 2. Let $A$ be a densely maximal monotone operator on $X \times X^{*}$ and $E \subset X^{*}$. Suppose that $\forall u^{*} \in E, \exists u \in X$ such that

$$
\inf _{\left(v, v^{*}\right) \in A}\left\langle v^{*}-u^{*}, v-u\right\rangle>-\infty .
$$

Then

$$
E \subset \overline{\mathcal{R}(A)} \quad \text { and } \quad \operatorname{int}(E) \subset \mathcal{R}\left(\bar{A}^{\tau}\right)
$$

Proof. - Let us prove that $E \subset \overline{\mathcal{R}(A)}$. For, let $u^{*} \in E$, and let us fix $\varepsilon>0$ sufficiently small. Then by [8, Theorem 4.1], for every $\lambda>0$ there exists $v_{\lambda} \in$ $\mathcal{D}(A), y_{\lambda}^{*} \in J_{\varepsilon} v_{\lambda}$ and $z_{\lambda}^{*} \in A v_{\lambda}$ such that

$$
u^{*}=\lambda y_{\lambda}^{*}+z_{\lambda}^{*} .
$$

By (1) there exist $u \in X$ and $c>0$ such that

$$
\forall\left(v, v^{*}\right) \in A \quad\left\langle v^{*}-u^{*}, v-u\right\rangle \geq-c .
$$

Let us take $v=v_{\lambda}$ and $v^{*}=z_{\lambda}^{*}=u^{*}-\lambda y_{\lambda}^{*}$; then for every $\lambda>0$

$$
\left\langle v^{*}-u^{*}, v-u\right\rangle=-\left\langle\lambda y_{\lambda}^{*}, v_{\lambda}-u\right\rangle \geq-c .
$$

According to Lemma 1, one has

$$
\left\langle y_{\lambda}^{*}, v_{\lambda}\right\rangle \geq\left\|y_{\lambda}^{*}\right\| \cdot\left\|v_{\lambda}\right\|-\varepsilon \quad \text { and } \quad\left\|y_{\lambda}^{*}\right\|^{2} \geq 2 \varepsilon+\left\|v_{\lambda}\right\|^{2},
$$


which imply that

$$
\begin{aligned}
c & \geq \lambda\left(\left\langle y_{\lambda}^{*}, v_{\lambda}\right\rangle-\left\langle y_{\lambda}^{*}, u\right\rangle\right) \\
& \geq \frac{\lambda}{2}\left(\left\|y_{\lambda}^{*}\right\|^{2}+\left\|v_{\lambda}\right\|^{2}-2 \varepsilon\right)-\frac{\lambda}{2}\left(\left\|y_{\lambda}^{*}\right\|^{2}+\|u\|^{2}\right) \\
& \geq \frac{\lambda}{2}\left(\left\|v_{\lambda}\right\|^{2}-\|u\|^{2}-2 \varepsilon\right) .
\end{aligned}
$$

We deduce that the family $\left\{\sqrt{\lambda}\left\|v_{\lambda}\right\|: \lambda>0\right\}$ is bounded for bounded $\lambda>0$. This yields $\lim _{\lambda \rightarrow 0^{+}}\left\|\lambda v_{\lambda}\right\|=0$ and in view of $\left\|\lambda y_{\lambda}^{*}\right\| \leq\left\|\lambda v_{\lambda}\right\|+\lambda \sqrt{2 \varepsilon}$, we derive

$$
\lim _{\lambda \rightarrow 0^{+}}\left\|\lambda y_{\lambda}^{*}\right\|=0 \text {. }
$$

Since $z_{\lambda}^{*} \in A v_{\lambda}$ (i.e., $\left.z_{\lambda}^{*} \in \mathcal{R}(A)\right)$ and $z_{\lambda}^{*}=u^{*}-\lambda y_{\lambda}^{*}$ strongly converges to $u^{*}$, it follows that $u^{*} \in \overline{\mathcal{R}(A)}$.

- Let us prove that $\operatorname{int}(E) \subset \mathcal{R}\left(\bar{A}^{\tau}\right)$. Let $u^{*} \in \operatorname{int}(E)$. Then there exists $r>0$ such that $u^{*}+r B^{*} \subset E$. Let $w^{*} \in r B^{*}$. Then, as a result of these facts and assumption (2), there exists $w \in X$ such that for every $\lambda>0$ we have

$$
\left\langle-\lambda y_{\lambda}^{*}+w^{*}, v_{\lambda}-w\right\rangle \geq-c
$$

since $z_{\lambda}^{*}=u^{*}-\lambda y_{\lambda}^{*} \in A v_{\lambda}$. Hence for some $\lambda_{0}>0$ we have $\left.\left.\forall \lambda \in\right] 0, \lambda_{0}\right]$

$$
\begin{aligned}
\left\langle w^{*}, v_{\lambda}\right\rangle & \leq c+\lambda\left(\left\langle y_{\lambda}^{*}, w\right\rangle-\left\langle y_{\lambda}^{*}, v_{\lambda}\right\rangle\right)+\left\langle w^{*}, w\right\rangle \\
& \leq c+\frac{\lambda}{2}\left(\|w\|^{2}-\left\|v_{\lambda}\right\|^{2}+2 \varepsilon\right)+\left\langle w^{*}, w\right\rangle \\
& \leq c+\frac{\lambda_{0}}{2}\left(\|w\|^{2}+2 \varepsilon\right)+\left\langle w^{*}, w\right\rangle .
\end{aligned}
$$

Thus $\forall w^{*} \in r B^{*}, \sup _{0<\lambda \leq \lambda_{0}}\left\langle w^{*}, v_{\lambda}\right\rangle<+\infty$. We conclude by using the uniform boundedness theorem that $\left\{v_{\lambda} ; 0<\lambda \leq \lambda_{0}\right\}$ is bounded in $X \subset X^{* *}$.

We can extract a subnet, also denoted by $\left\{v_{\lambda}\right\}$, such that $v_{\lambda} \rightarrow \bar{v} \in X^{* *}$ for the weak topology $\sigma\left(X^{* *}, X^{*}\right)$ whenever $\lambda \rightarrow 0^{+}$. We claim that $\bar{v} \in \mathcal{D}\left(\bar{A}^{\tau}\right)$ and $u^{*} \in \bar{A}^{\tau}(\bar{v})$. For, it suffices to show that

$$
\forall\left(v, v^{*}\right) \in A \quad\left\langle u^{*}-v^{*}, \bar{v}-v\right\rangle \geq 0 .
$$

To that end, let $\left(v, v^{*}\right) \in A$. Since $z_{\lambda}^{*} \in A v_{\lambda}$ and $A$ is monotone, we deduce

$$
\forall \lambda>0 \quad\left\langle z_{\lambda}^{*}-v^{*}, v_{\lambda}-v\right\rangle \geq 0 .
$$

Now, $\lim _{\lambda \rightarrow 0^{+}}\left\|z_{\lambda}^{*}-u^{*}\right\|=\lim _{\lambda \rightarrow 0^{+}}\left\|\lambda y_{\lambda}^{*}\right\|=0$ and $v_{\lambda} \rightarrow \bar{v} \in X^{* *}$ for $\sigma\left(X^{* *}, X^{*}\right)$ imply that

$$
\begin{aligned}
0 & \leq \liminf _{\lambda \rightarrow 0^{+}}\left\langle z_{\lambda}^{*}-v^{*}, v_{\lambda}-v\right\rangle \\
& \leq \limsup _{\lambda \rightarrow 0^{+}}\left\langle z_{\lambda}^{*}, v_{\lambda}\right\rangle-\liminf _{\lambda \rightarrow 0^{+}}\left\langle z_{\lambda}^{*}, v\right\rangle-\liminf _{\lambda \rightarrow 0^{+}}\left\langle v^{*}, v_{\lambda}\right\rangle+\left\langle v^{*}, v\right\rangle \\
& \leq\left\langle u^{*}-v^{*}, \bar{v}-v\right\rangle .
\end{aligned}
$$

It follows that $u^{*} \in \bar{A}^{\tau}(\bar{v})$, and thus $u^{*} \in \mathcal{R}\left(\bar{A}^{\tau}\right)$.

Theorem 1. Let $X$ be a general Banach space. Let $A_{1}$ and $A_{2}$ be two monotone operators satisfying

(i) $A_{1}$ and $A_{2}$ are $3^{*}$-monotones.

(ii) $A_{1}+A_{2}$ is densely maximal. 
Then

$$
\overline{\mathcal{R}\left(A_{1}+A_{2}\right)}=\overline{\mathcal{R}\left(A_{1}\right)+\mathcal{R}\left(A_{2}\right)}
$$

and

$$
\operatorname{int} \mathcal{R}\left(A_{1}+A_{2}\right) \subset \operatorname{int}\left(\mathcal{R}\left(A_{1}\right)+\mathcal{R}\left(A_{2}\right)\right) \subset \operatorname{int} \mathcal{R}\left(\overline{A_{1}+A_{2}}{ }^{\tau}\right) .
$$

Proof. It is obvious that

$\overline{\mathcal{R}\left(A_{1}+A_{2}\right)} \subset \overline{\mathcal{R}\left(A_{1}\right)+\left(\mathcal{R}\left(A_{2}\right)\right)} \quad$ and $\quad \operatorname{int} \mathcal{R}\left(A_{1}+A_{2}\right) \subset \operatorname{int}\left(\mathcal{R}\left(A_{1}\right)+\left(\mathcal{R}\left(A_{2}\right)\right)\right.$.

Let us establish the other ones. Define $A$ and $E$ of the last lemma by $A:=A_{1}+A_{2}$ and $E:=\mathcal{R}\left(A_{1}\right)+\mathcal{R}\left(A_{2}\right)$. All that needs to be shown is condition (1). For, let $u^{*} \in E$; then $u^{*}=u_{1}^{*}+u_{2}^{*}$ for $u_{i}^{*} \in \mathcal{R}\left(A_{i}\right)$. By $3^{*}$-monotonicity of $A_{i}$, we have for $u \in D\left(A_{1}\right) \cap D\left(A_{2}\right)$

$$
\inf _{\left(v, v^{*}\right) \in A_{i}}\left\langle v^{*}-u_{i}^{*}, v-u\right\rangle>-\infty \quad \text { for } i=1,2,
$$

which implies that

$$
\begin{aligned}
\inf _{\left(v, v^{*}\right) \in A}\left\langle v^{*}-u^{*}, v-u\right\rangle & =\inf _{v_{1}^{*}+v_{2}^{*} \in A_{1}(v)+A_{2}(v)}\left\langle\left(v_{1}^{*}-u_{1}^{*}\right)+\left(v_{2}^{*}-u_{2}^{*}\right), v-u\right\rangle \\
& \geq \inf _{\left(v, v_{1}^{*}\right) \in A_{1}}\left\langle\left(v_{1}^{*}-u_{1}^{*}\right), v-u\right\rangle+\inf _{\left(v, v_{2}^{*}\right) \in A_{1}}\left\langle\left(v_{2}^{*}-u_{2}^{*}\right), v-u\right\rangle \\
& >-\infty .
\end{aligned}
$$

This means condition (1) is satisfied. Hence the theorem is proven.

Remarks. (a) Let us remark that the $3^{*}$-monotonicity condition on $A_{1}$ and $A_{2}$ can be replaced by

$$
\forall u^{*} \in \mathcal{R}\left(A_{1}\right)+\mathcal{R}\left(A_{2}\right), \exists u \in X \quad \text { such that } \quad \inf _{\left(v, v^{*}\right) \in A_{1}+A_{2}}\left\langle v^{*}-u^{*}, v-u\right\rangle>-\infty .
$$

(b) The assertion of the main theorem remains true when we replace the $3^{*}$ monotonicity of $A_{1}$ by $D\left(A_{1}\right) \subset D\left(A_{2}\right)$.

Corollary 1. Suppose that $X$ is a reflexive Banach space. Let $A_{1}$ and $A_{2}$ be two $3^{*}$-monotone operators satisfying that $\overline{A_{1}+A_{2}}$ is maximal. Then

$$
\operatorname{int} \mathcal{R}\left(\overline{A_{1}+A_{2}}\right)=\operatorname{int}\left(\mathcal{R}\left(A_{1}\right)+\mathcal{R}\left(A_{2}\right)\right) \quad \text { and } \quad \overline{\mathcal{R}\left(A_{1}+A_{2}\right)}=\overline{\mathcal{R}\left(A_{1}\right)+\mathcal{R}\left(A_{2}\right)} \text {. }
$$

The proof is immediate, since when the space $X$ is reflexive and $A=\overline{A_{1}+A_{2}}$ is maximal monotone, $\bar{A}^{\tau}=A$.

Corollary 2. Let $f$ and $g$ be two proper convex lower semicontinuous functions defined from a Banach space $X$ in $\mathbb{R} \cup\{+\infty\}$. Assume that

$$
\bigcup_{t>0} t(\operatorname{dom}(f)-\operatorname{dom}(g)) \text { is a closed linear subspace of } X \text {. }
$$

Then

$$
\overline{\mathcal{R}(\partial f)+R(\partial g)}=\overline{\mathcal{R}(\partial(f+g))} \quad \text { and } \quad(\mathcal{R}(\partial f)+\mathcal{R}(\partial g))=\operatorname{int} \mathcal{D} \partial\left(f^{*}+g^{*}\right) .
$$

Proof. Using [1, Corollary 1] we have $\partial(f+g)=\partial f+\partial g$. Rockafellar's theorem in [16] guarantees that $\partial f$ and $\partial g$ are cyclic monotone, in particular, $3^{*}$-monotone. We have

$$
\overline{\mathcal{R}(\partial f)+\mathcal{R}(\partial g)}=\overline{\mathcal{R}(\partial f+\partial g)}=\overline{\mathcal{R}(\partial(f+g))}
$$


and

$$
\operatorname{int}(\mathcal{R}(\partial f)+\mathcal{R}(\partial g))=\operatorname{int} \mathcal{R}\left(\overline{\partial(f+g)}^{\tau}\right) .
$$

Attouch and Brézis [1, Theorem 1] show that under assumption (4)

$$
(f+g)^{*}=f^{*} \stackrel{e}{+} g^{*} .
$$

Therefore

$$
{\overline{\partial(f+g)^{\tau}}}^{\tau}=\left(\partial(f+g)^{*}\right)^{-1}=\left(\partial\left(f^{*} \stackrel{e}{+} g^{*}\right)\right)^{-1},
$$

which completes the proof.

\section{REFERENCES}

1. H. Attouch and H. Brézis, Duality for the sum of convex functions in general Banach spaces, Aspects of mathematics and its applications (J. B. Arroso, ed.), North-Holland, Amsterdam, 1986, pp. 125-133. MR 87m:90095

2. J.-P. Aubin and I. Ekeland, Applied nonlinear analysis, Wiley, New York, 1984. MR 87a:58002

3. H. Brézis and A. Haraux, Image d'une somme d'opérateurs monotones et applications, Israel J. Math. 23 (1976), 165-186. MR 53:3803

4. H. Brézis and L. Nirenberg, Characterization of the ranges of some nonlinear operators and applications to boundary value problems, Ann. Scuola Norm. Sup. Pisa CI. Sci. Ser. IV 5 (1978), 225-326. MR 58:23813

5. F. Browder, On the principle of H. Brézis and its applications, J. Funct. Anal. 25 (1977), 356-365. MR 56:3686

6. _ Image d'un opérateur maximal monotone et le principe de Landesman-Lazer, C. R. Acad. Sci. Paris Sér. A 287 (1978), 715-718. MR 80b:47069

7. B. Calvert and C. Gupta, Nonlinear elliptic boundary value problems in $L^{p}$ space and sums of ranges of accretive operators, Nonlinear Anal. T.M.A. 2 (1978), 1-26. MR 80i:35083

8. J.-P. Gossez, Opérateurs monotones non linéaires dans les espaces de Banach non réflexifs, J. Math. Anal. Appl. 34 (1971), 371-395. MR 47:2442

9. - On the range of a coercive maximal monotone operator in a nonreflexive Banach space, Proc Amer. Math. Soc. 35 (1972), 88-92. MR 45:7544

10. On the extensions of the bidual of a maximal monotone operator, Proc. Amer. Math. Soc. 62 (1977), 67-71. MR 55:1150

11. C. Gupta and P. Hess, Existence theorems for nonlinear noncoercive operator equations and nonlinear elliptic boundary value problems, J. Differential Equations 22 (1976), 305-313. MR 57:13600

12. A. G. Kartsatos, Mapping theorems involving ranges of sums on nonlinear operators, Nonlinear Anal. T.M.A. 6 (1982), 271-278. MR 83k:47036

13. C. H. Morales, On the range of sums of accretive and continuous operators in Banach spaces, Nonlinear Anal. T.M.A. 19 (1992), 1-9. MR 93d:47096

14. R. R. Phelps, Lectures on maximal monotone operators, Lectures given at Prague-Paseky Summer School, 1993.

15. S. Reich, The range of sums of accretive and monotone operators, J. Math. Anal. Appl. 68 (1979), 310-317. MR 80g:47060

16. R. T. Rockafellar, On the maximal monotonicity of subdifferential mappings, Pacific J. Math. 33 (1970), 209-216. MR 41:7432

17. E. Zeidler, Nonlinear functional analysis and applications II-B: Nonlinear monotone operators, Springer-Verlag, New York, 1990. MR 91b:47002

Semlalia Faculty of Sciences, Mathematics, University Cadi Ayyad, Boulevard My Abdellah, B.P.S. 15, 40000 Marrakesh, Morocco 